\title{
Movilidad y dificultades del trabajo a distancia en tiempos de Covid-19: una visión de los alumnos de la Licenciatura en Música del Instituto de Artes de la Universidad Autónoma del Estado de Hidalgo
}

\author{
Distance learning and mobility challenges during the Covid-19 outbreak: a view of the music \\ students from the Arts Institute of the Autonomous University of Hidalgo State
}

Mauricio Hernández-Monterrubio ${ }^{1}$, Rosalía Trejo-León ${ }^{2}$, Raúl Cortés-Cervantes ${ }^{3}$

\begin{abstract}
:
This paper covers five topics from an investigation carried out with students enrolled in the Bachelor of Music from the Arts Institute of the Autonomous University of Hidalgo State. The test "Educational experiences during COVID-19 outbreak" was applied during the first period of the health contingency in Mexico (April 2020). The answers of 93 students to five questions are related to: 1) their geographical mobility, 2) their age, 3) the musical instruments they study and the relation with the mobility impact, 4) their learning challenges during COVID-19 outbreak and, 5) the problems faced to do academic activities. The answers reflect some real situations students had during the first half of 2020 and maybe will continue to the second half of the year due to the COVID-19 outbreak. Through a systematic analysis, the research aims to provide tools to improve institutional and academic strategies to solve the lack of preparation for facing health crisis in higher education music institutions.
\end{abstract}

Keywords:

bachelor of music, covid-19, school difficulties, distance learning, geographical mobility

\section{Resumen:}

En este artículo se exponen cinco tópicos de una investigación realizada con alumnos de la Licenciatura en Música del Instituto de Artes (IA) UAEH. Se aplicó un cuestionario durante el primer periodo de la contingencia sanitaria en México (abril 2020), llamado "Experiencias educativas durante la emergencia sanitaria por Covid-19". En esta ocasión se presentan las respuestas de 93 alumnos del IA a cinco cuestiones relacionadas con: 1) la movilidad geográfica que tuvieron los alumnos, 2) la edad de la población estudiantil encuestada, 3) los instrumentos musicales que estudian con relación al impacto de esa movilidad, 4) situaciones que dificultaron sus actividades escolares y 5) de qué tipo fueron estos problemas para realizar el trabajo académico a distancia. Las respuestas reflejan algunas situaciones reales que los alumnos enfrentaron durante dicho periodo y que, probablemente, todavía pueden estar viviendo con la prolongación de la contingencia durante el segundo semestre del año 2020. A través de un análisis sistemático, el estudio pretende dar herramientas para mejorar estrategias institucionales y académicas que ayuden a solventar la falta de preparación para enfrentar la crisis sanitaria en el ámbito de la Educación Superior en el área de música.

\section{Palabras Clave:}

licenciatura en música, covid-19, dificultades escolares, trabajo a distancia, movilidad geográfica

\footnotetext{
1 *Autor de correspondencia. Instituto de Artes, Universidad Autónoma del Estado de Hidalgo, https://orcid.org/0000-00021916-8271, mauricio_hernandez5904@uaeh.edu.mx

2 Instituto de Artes, Universidad Autónoma del Estado de Hidalgo, https://orcid.org/0000-0002-8001-0844, rosalia_trejo@uaeh.edu.mx

${ }^{3}$ Instituto de Artes, Universidad Autónoma del Estado de Hidalgo, https://orcid.org/0000-0001-6383-1854, raul_cortes6138@uaeh.edu.mx
} 


\section{INTRODUCCIÓN}

El siguiente artículo está desarrollado con base en un cuestionario realizado a estudiantes y maestros del área de música del Instituto de Artes (IA) de la Universidad Autónoma del Estado de Hidalgo (UAEH). Los datos que se presentan forman parte de las respuestas que se obtuvieron del cuestionario de los estudiantes. En este trabajo se expone parte de la realidad que los alumnos del Instituto de Artes pasaron en el periodo comprendido de marzo a julio de 2020, debido a la contingencia sanitaria por Covid-19, que abarcó casi la totalidad del primer semestre educativo del año. Este estudio ha arrojado importantes resultados que se analizan en profundidad para comprender las problemáticas que se presentan a los alumnos para seguir con sus estudios universitarios.

Para en marcar este trabajo, se desarrolló una exploración de textos que abordan el tema de las dificultades de la educación superior en México durante el paso del Covid19. El primero trata el tema del hogar como un espacio donde se desarrollan actividades yuxtapuestas. Si bien, ahora tras la emergencia sanitaria la casa se ha vuelto el centro de todas las acciones, no podemos pasar desapercibido algu nos problemas que son palpables en los hogares del país. Como lo menciona De la Cruz (2020), hay situaciones que afectan de manera importante las tareas académicas en casa, ya que estando en ella, los alumnos son llamados a realizar otros trabajos que pueden generar falta de tiempo $u$ organización para sus deberes escolares. La mayoría de los hogares no cuentan con los espacios adecuados ni con los recursos tecnológicos idóneos. En muchos casos hay violencia intrafamiliar que afecta directamente a los jóvenes. El apoyo familiar es escaso y los alumnos no están capacitados para llevar una agenda de actividades en línea por ellos mismos.

Lo anteriormente citado también es comentado por López y Rodríguez (2020), quienes además mencionan que a pesar de que a esta generación se les conoce como la "generación tecnológica", muchos de ellos carecen de habilidades para realizar tareas de forma virtual. Estas circunstancias pueden llevar a un desfase de conocimientos entre quienes entienden más la tecnología y quienes no.

Otro punto de interés es la posición de las instituciones de educación superior frente a la pandemia. La instrucción virtual ha recibido mayor atención que la presencial, llegando esta última a una parálisis. Las instituciones por mucho tiempo han mantenido una forma de organización, así como las mismas estrategias de trabajo, que según Chehaibar (2020) "se evidencian anquilosadas y rígidas para dar cauce a nuevas y renovadas rutas de actuación ante las circunstancias de aislamiento físico que esta pandemia ha impuesto" ( $p$. 84). Además, la autora realiza una reflexión no solamente de la rigurosidad de las instituciones educativas en los formatos de trabajo y su funcionalidad arraigada a la modalidad presencial, sino que desarrolla un análisis global de otros efectos colaterales de la pandemia en la escala social, afirmando lo siguiente:

El "Gran Confinamiento" y la globalidad de esta enfermedad, sumada a las condiciones de interconectividad planetaria (por medio de las redes sociales y los diversos medios tecnológicos) han puesto en evidencia una serie de elementos que, quizá, no habían sido reflexionados a profundidad. Entre ellos podemos señalar la prueba flagrante de las desigualdades socioeconómicas y los rasgos de incertidumbre del mundo en que vivimos, así como las probabilidades de que la presencia de enfermedades altamente contagiosas (como la covid-19) vaya a cambiar la forma en que llevamos a cabo y entendemos aspectos de la cotidianidad que pensábamos establecidos y constantes, como pueden ser el transporte público, el trabajo, las comunicaciones, el comercio, los espectáculos y la educación". (Chehaibar, 2020, p.84).

Lo citado refleja la problemática que desemboca la crisis sanitaria, que rodea las actividades escolares y que afecta directamente a los procesos educativos. No se 
trata únicamente de realizar las clases en línea, sino de impulsar una planeación incluyente. Así pues, hace falta que las universidades hagan inversiones importantes en conectividad, equipamiento y forme a sus académicos correctamente. $Y$, al mismo tiempo, deben considerar las situaciones sociales, económicas y afectivas que se viven con la pandemia. En este sentido, Chehaibar (2020) comenta que:

Se ha insistido en cumplir el programa, en hacer actividades en casa que estén "alineadas" al currículo, como si el único sentido de educar fuera dar cuenta del contenido prescrito en el plan y los programas de estudio que, además, debe ser documentado en "Carpetas de experiencias" que permitan calificar a los maestros para "salvar el año escolar". (p.87).

Esto indica que la educación en México se ha preocu pado por terminar los contenidos establecidos por el cu rrículum y que, ante esta emergencia sanitaria, solo importa terminar el semestre en lugar de dar mejores herramientas de aprendizaje a los alumnos.

Por último, cabe mencionar que, en nuestro país, el acceso a la tecnología o el uso de esta ha llevado un proceso lento y desigual en los diversos estratos sociales, así como la capacitación para los integrantes de instituciones educativas. A este respecto, Barrón (2020) afirma que:

Los desafíos y los retos no han sido menores, y son de diversa índole, ya sean de corte tecnológico o de la formación de los docentes y de los estudiantes para el uso y manejo de las plataformas digitales. Además, debe considerarse que en nuestro país 60 por ciento de la población carece de una computadora y no tiene acceso a internet, y quien cuenta con éste, el ancho de banda y la conectividad son limitados para el trabajo intenso que se requiere. (p.68).

Las referencias a estos autores ofrecen un marco inicial de la realidad que se vive en nuestro país. Es claro que en México se carece de infraestructura y equipamiento para atender debidamente las clases en línea. A ello se vinculan problemáticas como la conectividad estable a internet, violencia intrafamiliar y la dificultad para tener un espacio cómodo en los hogares. Éstas son realidades que deben ser atendidas para ofrecer las mejores soluciones a las situaciones que vivimos hoy en día.

\section{DESARROLLO}

El cuestionario fue enviado a los alumnos de los diez semestres de la Licenciatura en Música del Instituto de Artes por medio de sus tu tores académicos. El enlace con el formulario de Google fue diseñado con el título "Experiencias educativas durante la emergencia sanitaria por Covid-19 en el Área de Música del Instituto de Artes de la UAEH”. En él fueron expuestas dieciséis preguntas, a las cuales respondieron 93 estudiantes de diversos instrumentos musicales.

En este trabajo se realizó un recorte de la primera y segunda sección del formulario que muestran la identificación de los participantes y sus condiciones académicas en la modalidad virtual. Se agruparon cinco tópicos de análisis destacando los siguientes aspectos: 1 ) identificar en qué municipio y estado estuvieron resguardados los alumnos durante los primeros cuatro meses de la contingencia, 2) cuál es su edad, 3) qué instrumento estudian, 4) si tuvieron situaciones que dificultaron sus actividades escolares y 5) de qué tipo fueron estos problemas para realizar el trabajo académico a distancia. Las respuestas reflejan situaciones reales en que los alumnos se encontraron durante dicho periodo, y que probablemente todavía pueden vivir con la prolongación de la contingencia durante el segundo semestre del año 2020.

Con relación al primer tópico, el $20.43 \%$ de los estudiantes permanecieron la mayor parte del tiempo resguardados en la ciudad de Pachuca, el $7.53 \%$ en Tulancingo, el 6.45\% en Mineral del Monte, el 5.38\% en Mineral de la Reforma y el resto en 35 diferentes localidades del Estado de Hidalgo, o Estados aledaños, como se muestra en la Tabla 1. 


\begin{tabular}{|c|c|c|}
\hline Localidad & Alumnos & Porcentaje \\
\hline Actopan & 1 & $1.08 \%$ \\
\hline Ajacuba & 1 & $1.08 \%$ \\
\hline Alfajayucan & 1 & $1.08 \%$ \\
\hline Atizapán & 1 & $1.08 \%$ \\
\hline Atotomoc & 1 & $1.08 \%$ \\
\hline Atotonilco & 1 & $1.08 \%$ \\
\hline Cardonal & 1 & $1.08 \%$ \\
\hline CDMX & 3 & $3.23 \%$ \\
\hline Ciudad Ixtepec & 1 & $1.08 \%$ \\
\hline Ciudad Sahagún & 3 & $3.23 \%$ \\
\hline $\begin{array}{l}\text { Cuautepec de } \\
\text { Hinojosa }\end{array}$ & 1 & $1.08 \%$ \\
\hline Francisco I. Madero & 2 & $2.15 \%$ \\
\hline Huejutla & 1 & $1.08 \%$ \\
\hline Ixtapaluca & 1 & $1.08 \%$ \\
\hline $\begin{array}{l}\text { Mineral de la } \\
\text { Reforma }\end{array}$ & 5 & $5.38 \%$ \\
\hline Mineral del Monte & 6 & $6.45 \%$ \\
\hline Naucalpan & 1 & $1.08 \%$ \\
\hline Localidad & Alumnos & Porcentaje \\
\hline No especificado & 8 & $8.60 \%$ \\
\hline Otumba & 1 & $1.08 \%$ \\
\hline Pachuca & 19 & $20.43 \%$ \\
\hline Papantla & 1 & $1.08 \%$ \\
\hline Progreso de Obregón & 1 & $1.08 \%$ \\
\hline $\begin{array}{l}\text { San Agustín } \\
\text { Zapotlán }\end{array}$ & 1 & $1.08 \%$ \\
\hline San Felipe Hidalgo & 1 & $1.08 \%$ \\
\hline $\begin{array}{l}\text { San Martin de las } \\
\text { Pirámides }\end{array}$ & 1 & $1.08 \%$ \\
\hline San Salvador & 1 & $1.08 \%$ \\
\hline Temascalapa & 3 & $3.23 \%$ \\
\hline Teotihuacan & 1 & $1.08 \%$ \\
\hline Tepeapulco & 2 & $2.15 \%$ \\
\hline Tepetitlán & 1 & $1.08 \%$ \\
\hline Tizayuca & 3 & $3.23 \%$ \\
\hline Tlalnepantla & 5 & $5.38 \%$ \\
\hline Tlanalapa & 1 & $1.08 \%$ \\
\hline Tolcayuca & 1 & $1.08 \%$ \\
\hline Tulancingo & 7 & $7.53 \%$ \\
\hline Xicotepec & 1 & $1.08 \%$ \\
\hline Zacatecas & 1 & $1.08 \%$ \\
\hline Zacatlán & 1 & $1.08 \%$ \\
\hline Zapotlán & 1 & $1.08 \%$ \\
\hline TOTAL & 93 & $100.00 \%$ \\
\hline
\end{tabular}

de Hidalgo, aunque también se cuenta con alumnos del Estado de México, Puebla, la CDMX, Veracruz, Tlaxcala, Oaxaca y Zacatecas. Esto se ejemplifica en la Figura 1.

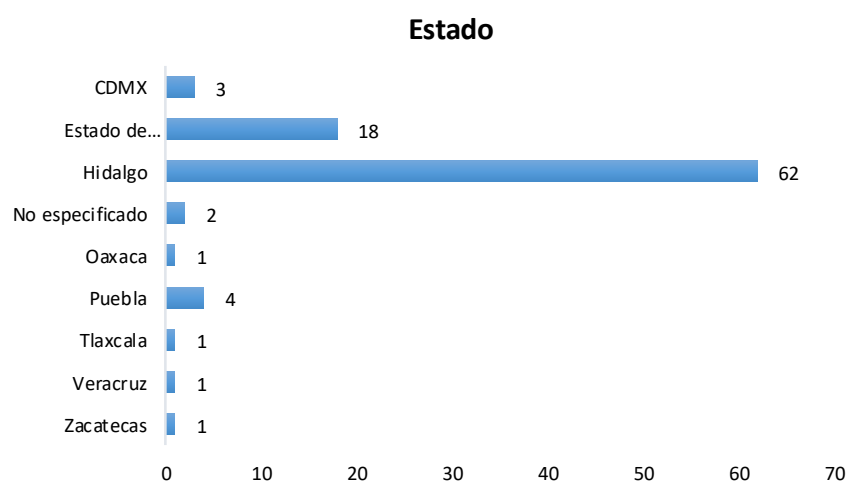

Figura 1. Población estudiantil del IA por Estados

La tabla 1 y la figura 1 muestran que los estudiantes tuvieron una movilidad a diferentes localidades del Estado de Hidalgo y estados aledaños. Ello significa que la mayoría no permaneció cerca del centro de estudios, localizado en Mineral del Monte.

La Licenciatura en Música concentra a una población de alumnos que son mayores de edad; algunos de ellos ya viven con recursos propios y otros son apoyados económicamente por su familia. Este hecho arroja retos económicos y sociales que deben ser atendidos al mismo tiempo junto con las exigencias de la licenciatura.

El $20.43 \%$ de los alumnos encuestados manifestaron tener 22 años, el 13.98\% mencionó 20 años y el mismo porcentaje fue obtenido en el grupo que manifestó tener 24 años, el $12.90 \%$ tiene 21 años, el 10.75 tiene 23 años, el $6.45 \%$ tiene 19 años y el $21.51 \%$ supera los 24 años. La Figura 2 muestra la cantidad de respuestas con relación a los rangos de edad en las que más oscila la población estudiantil.

Tabla 1. Localidades de permanencia de los alumnos en los primeros cuatro meses de contingencia

Esto significa que la mayor población estudiantil del área de Música del IA está integrada por alumnos del Estado 


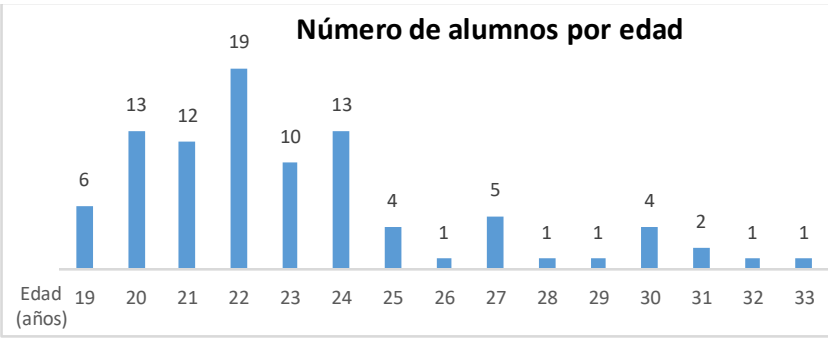

Figura 2. Edad específica de los alumnos encuestados

Esto nos indica que el $78.49 \%$ de los alumnos encuestados está en un rango de edad que oscila de los 19 a los 24 años. Este dato es congruente cuando se considera que la Licenciatura en Música cuenta con un curso propedéutico de 1 año, por consiguiente, los alumnos recién egresados de preparatoria ingresan al primer semestre de la licenciatura cuando tienen en promedio 19 años.

Los alumnos eligen un solo instrumento musical al inicio de sus estudios para convertirse en interpretes profesionales; la elección se encuentra dentro de una gama de 16 (UAEH, 2019). Fue pertinente realizar un análisis de esa elección. Esta categoría se organizó por familias de instrumentos. De la familia de cuerdas el $40.86 \%$ de los alumnos estudia algún instrumento como violín, viola, violonchelo o contrabajo. De los alientos madera y metales (como flauta, clarinete, oboe, fagot, trompeta, trombón y tuba) reúnen el $18.28 \%$. A estos le siguen los alumnos que estudian canto con $12.90 \%$, guitarra con $11.83 \%$, piano con $9.68 \%$ y percusiones con $5.38 \%$.

Estos datos indican que casi el $85 \%$ de los estudiantes se especializa en un instrumento que puede ser adquirido con relativa facilidad porque su costo no es exageradamente elevado para la mayoría. Solo el piano y algunos de los instrumentos de percusión como la marimba, xilófono o los timbales son de precio elevado y, por lo tanto, no resultan de fácil adquisición. En estos casos, los estudiantes comúnmente realizan sus prácticas dentro de las instalaciones del Instituto. Sin embargo, frente a la contingencia sanitaria deben solicitar el permiso correspondiente a las autoridades para hacer uso de dichos instrumentos. En la Tabla 2 se muestra la cantidad de alumnos encuestados que estudian instrumentos de cada familia y los porcentajes en relación con el total de respuestas obtenidas.

\begin{tabular}{|l|l|l|}
\hline Familia de instrumentos & Alumnos & Porcentaje \\
\hline Alientos madera & 9 & $9.68 \%$ \\
\hline Alientos metal & 8 & $8.60 \%$ \\
\hline Canto & 12 & $12.90 \%$ \\
\hline Cuerdas & 38 & $40.86 \%$ \\
\hline Guitarra & 11 & $11.83 \%$ \\
\hline No contestó & 1 & $1.08 \%$ \\
\hline Percusiones & 5 & $5.38 \%$ \\
\hline Piano & 9 & $9.68 \%$ \\
\hline
\end{tabular}

Tabla 2. Alumnos con relación a la familia de instrumentos que estudian

La mayoría de los instrumentos que adquieren los alumnos pueden ser trasladados con facilidad; sin embargo, éstos deben practicarse en lugares adecuados a la sonoridad de cada uno, con espacios preparados acústicamente. En este periodo de pandemia, los estudiantes se movilizaron junto con su instrumento de estudio para resguardarse con sus familias. Desafortunadamente, los hogares no cuentan con esos lugares preparados para las prácticas instrumentales. Además, el tiempo de dedicación para esas prácticas puede ir de 2 hasta 6 horas por día; esto puede llegar a ser molesto para los familiares con quien cohabita el estudiante y generar estrés en el ambiente.

Cuando se cuestionó sobre las situaciones que dificultaron la atención de sus actividades escolares a distancia, el $72 \%$ mencionó que sí tuvieron dificultades en este periodo, y el $28 \%$ mencionó que no. Como se observa en la Figura 3, los encuestados marcaron el tipo de dificultades enlistadas, pudiendo escoger varias opciones. 


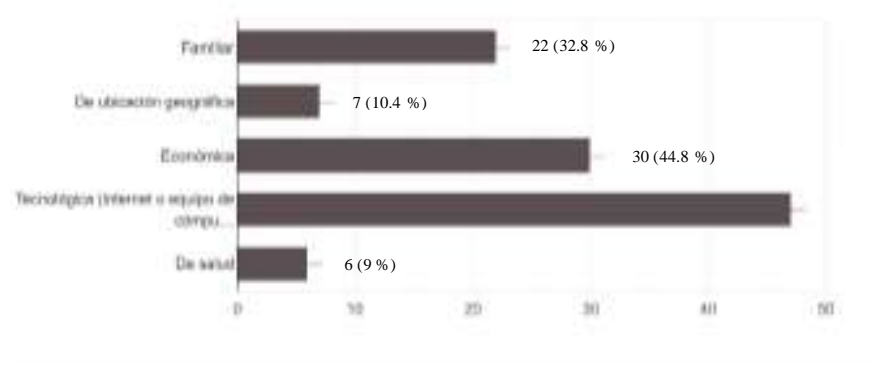

Figura 3. Situaciones que dificultaron la atención académica de los alumnos

Así mismo, los alumnos mencionaron tener otras dificultades, tales como: la falta de instrumento, el nulo aprendizaje, la falta de espacios adecuados para estudiar, la complicación de compartir el único equipo de cómputo que tienen en casa con sus hermanos, tenerque ir a trabajar por falta de recursos económicos, el poco contacto con sus maestros en las materias prácticas, así como problemas de concentración y ansiedad.

Cabe destacar que la mayor dificultad que se hizo presente fue la tecnológica (70.1\%), que incluye la falta de equipo o el acceso a internet. En segundo plano, se encuentra la dificu ltad económica (44.8\%) ya que muchas familias se quedaron sin ingresos por lo que los alumnos tuvieron que apoyar trabajando o en labores extras dentro del hogar, lo cual se conecta con el tercer aspecto mayormente mencionado, el aspecto familiar (32.8 \%).

\section{CONCLUSIONES}

En este trabajo se mostraron las respuestas a cinco de las 16 preguntas realizadas en el cuestionario a los alumnos del Instituto de Artes de la UAEH en el periodo de contingencia por Covid-19. Los resultados muestran dificultades por las que atraviesan los alumnos en esta situación emergente y por la cual tuvieron que improvisar procesos de estudio con sus instrumentos, cambiaron hábitos en su vida cotidiana, e inclusive tuvieron la necesidad de trasladarse a sus lugares de origen en otros municipios o Estados.

Dicha movilidad ha ocasionado una separación de los espacios educativos; Ios alu mnos han tenido que romper rutinas de estudio que los aleja de sus deberes académicos en marcados dentro de horarios establecidos por el currículo presencial en la universidad. Esto, a su vez, ha generado un distanciamiento de los lugares adecuados acústicamente para la práctica musical prolongada, se suspendió el acceso a espacios educativos como bibliotecas o salas de cómputo y, asimismo, ha dificultado la práctica de instrumentos como el piano o las percusiones que no pueden trasladarse, ya que son propiedad de la institución.

En cuanto al ámbito tecnológico, se observa que la movilidad a localidades alejadas de zonas urbanas afectó el trabajo académico por la falta de servicio de intern et o de recursos para acceder a él, tal como lo señalan otros estudios realizados en el país (Chehaibar, 2020; Barrón, 2020). En el caso de este cuestionario, éste fue el primer aspecto mencionado en la dificultad para continuar con las labores académicas a distancia.

Así también, podemos mencionar que la población estudiantil cuenta con la mayoría de edad. Esto no significa que los alumnos sean autónomos económicamente. Sin embargo, vivir alejados de sus familias les da independencia en su estilo de vida, donde sus actividades son organizadas en tiempos y espacios propios. Esta relativa independencia fue afectada con el regreso a la casa de sus familiares donde se mezclaron situaciones emocionales (por romper esa au tonomía), por los hábitos de estudio (sin horarios establecidos por una carga horaria oficial), y por actividades familiares espontáneas. Este último aspecto se encuentra enfatizado en la bibliografía explorada, que habla sobre el hogar convertido (de un momento a otro) en el único espacio para realizar todas las actividades cotidianas (De la Cruz, 2020). A pesar de que este artículo no trata a profundidad detalles sobre el contexto familiar, se destaca que este fue el tercer aspecto mayormente mencionado en las dificultades que afectaron el desarrollo de actividades académicas.

La segunda dificultad presentada en los encuestados fue el factor económico, ya que muchos jefes de familia perdieron sus empleos y los alumnos tuvieron que dejar de estudiar para ayudar y llevar sustento a sus familias (ver Figura 3). 
Los datos presentados aquí seguirán siendo profundizados en otros análisis, ya que el cuestionario completo contó con preguntas relacionadas a qué dispositivos tecnológicos fueron más utilizados para el envío de evidencias académicas, qué respuesta y comunicación tuvieron con sus profesores o incluso cómo observan este periodo dentro de su formación profesional entre otros tópicos que serán explorados en próximos artículos.

Así mismo se realizará un seguimiento a algunas cuestiones específicas del periodo extendido de la pandemia, diseñando nuevas herramientas de recolección de información, utilizando técnicas como la entrevista a profundidad o narrativas de los estudiantes de música. Este texto plantea un primer panorama de la situación, sin embargo, no pretende dar soluciones inmediatas. Con próximos análisis, es posible sugerir acciones pedagógicas para mejorar los procesos académicos a distancia en medio de este contexto de incertidumbre social.

\section{REFERENCIAS}

[1] Barrón Tirado, M. C. (2020). La educación en línea. Transiciones y disrupciones. En H. Casanova Cardiel (Coord.), Educación y pandemia: una visión académica (pp. 66-74). Ciudad de México: Universidad Nacional Autónoma de México, Instituto de Investigaciones sobre la Universidad y la Educación.

[2] Chehaibar, L. M. (2020). Flexibilidad curricular. Tensiones en tiempos de pandemia. En H. Casanova Cardiel (Coord.), Educación y pandemia: una visión académica (pp. 83-91). Ciudad de México: Universidad Nacional Autónoma de México, Instituto de Investigaciones sobre la Universidad y la Educación.

[3] De la Cruz Flores, G. (2020). El hogar y la escuela: lógicas en tensión ante la COVID-19. En H. Casanova Cardiel (Coord.), Educación y pandemia: una visión académica (pp. 39-46). Ciudad de México: Universidad Nacional Autónoma de México, Instituto de Investigaciones sobre la Universidad y la Educación.

[4] López Ramírez, M., y Rodríguez, S. A. (2020). Trayectorias escolares en la educación superior ante la pandemia ¿continuar, interrumpir o desistir? En H. Casanova Cardiel (Coord.), Educación y pandemia: una visión académica (pp. 103-108). Ciudad de México: Universidad Nacional Autónoma de México, Instituto de Investigaciones sobre la Universidad y la Educación.
[5] Universidad Autónoma del Estado de Hidalgo, UAEH. (2019). Proyecto de Diseño Curricular de la Licenciatura en Música, México. 